\title{
Genetic diversity and phylogeny of Wolbachia in lepidopteran hosts
}

\author{
M. Yudina ${ }^{1,2 *}$, V. Dubatolov ${ }^{3}$, R. Bykov ${ }^{1}$, I. Mazunin ${ }^{4}$, Yu. Ilinsky ${ }^{1,2}$ \\ ${ }^{1}$ Institute of Cytology and Genetics SB RAS, Novosibirsk, Russia \\ ${ }^{2}$ Novosibirsk State University, Novosibirsk, Russia \\ ${ }^{3}$ Institute of Systematics and Ecology of Animals SB RAS, Novosibirsk, Russia \\ ${ }^{4}$ Immanuel Kant Baltic Federal University, Kaliningrad, Russia \\ *e-mail:Judina@bionet.nsc.ru
}

Key words: Wolbachia, Lepidoptera, multilocus sequence typing, forest pests

Motivation and Aim: Wolbachia are maternally inherited intracellular symbionts of arthropods and some nematodes. The genetic diversity of Wolbachia is subdivided into 16 supergroups (phyletic lines). Significant number of insect families appears to be unexplored for Wolbachia symbionts. Seventeen families of Lepidoptera are known to be infected with Wolbachia, while this order includes up to 200 families. Here we try to find Wolbachia infection in hosts of poorly investigated lepidopteran families and further analyse genetic data of Wolbachia isolates.

Methods and Algorithms: The insect collection consisted of 519 samples and included 255 species of 23 lepidopteran families. Some of these species are dangerous forest pests. The DNA extraction was individually performed from each specimen. Infection status was determined by PCR with Wolbachia specific primers targeted to the wsp and 16SrRNA loci. Genetic diversity of Wolbachia was determined by the multilocus sequence typing (MLST) protocol [1], which included five loci. Phylogenetic analysis was conducted using MEGA 6 [2].

Results: Wolbachia symbionts were found in 36 species of 12 lepidopteran families. Some of these species were forest pests. We found both new haplotypes and previously described Wolbachia variants. Most of symbiont haplotypes belonged to supergroup B, and remaining haplotypes belonged to supergroup A.

Conclusion: We present comprehensive results of Wolbachia incidence and Wolbachia genetic diversity in Lepidoptera. The results of this study are necessary to develop Wolbachia-based biotechnological methods of pest control.

Acknowledgements: Supported by the RFBR (16-04-00980, 18-316-00099).

\section{References}

1. Baldo L. et al. (2006) Multilocus sequence typing system for the endosymbiont Wolbachia pipientis. Applied Environmental Microbiology. 72(11):7098-7110.

2. Tamura K. et al. (2013) MEGA6: Molecular Evolutionary Genetics Analysis version 6.0. Molecular Biology Evolution. 30:2725-2729. 